\title{
ON SOME EXTREMAL PROPERTIES OF TRIGONOMETRIC POLYNOMIALS WITH REAL ROOTS
}

\author{
BY J. GERONIMUS
}

1. Introduction. L. Fejér [1],† O. Szász [2], [3], [4] and E. v. Egerváry [5] have found many interesting extremal properties of non-negative trigonometric polynomials. In particular, Szász [3] has found that for every non-negative trigonometric polynomial of order $\leqq n$ with real coefficients,

(1) $G_{n}(\theta)=1+R \sum_{k=1}^{n} \bar{\gamma}_{k} e^{i k \theta}, \quad\left(\gamma_{k}=\alpha_{k}+i \beta_{k} ; k=1,2, \cdots, n\right)$, the inequality

$$
\left|\gamma_{k}\right| \leqq 2 \cos \frac{\pi}{\left[\frac{n}{k}\right]+2}, \quad(k=1,2, \cdots, n)
$$

is valid. $\ddagger$

The object of this note is to find the minimum of the modulus of the first coefficient $\gamma_{n}$, supposing that all roots of $G_{n}(\theta)$ are real. The first problem of this kind has been considered by Blumenthal [6]; we shall return in $\$ 4$ to his problem and its generalization.

2. Equality of Roots of $G_{n}^{*}(\theta)$ for Problem 1 . Consider the following problem.

Problem 1. Find the minimum of the modulus of the first coefficient $\gamma_{n}$ of a non-negative trigonometric polynomial

$$
G_{n}(\theta)=1+R \sum_{k=1}^{n} \bar{\gamma}_{k} e^{i k \theta}
$$

of order $n$ with real roots.

$\dagger$ Numbers in brackets refer to the Bibliography at the end.

$\ddagger \mathcal{R} z$ means real part of $z ;[a]$ means the greatest integer $\leqq a$. 
In order to solve this problem we shall prove the following simple lemma.

Lemma 1. All roots of the polynomial $G_{n}{ }^{*}(\theta)$ for which the minimum in Problem 1 is attained must be equal.

Consider a non-negative trigonometric polynomial $\dagger$

(3) $G_{n}(\theta)=4 \sin ^{2} \frac{\theta-\theta_{1}}{2} \sin ^{2} \frac{\theta-\theta_{2}}{2} F_{n-2}(\theta)=R \sum_{k=0}^{n} \bar{\gamma}_{k} e^{i k \theta}$,

where $F_{n-2}(\theta)$ is a non-negative trigonometric polynomial of order $n-2$ with real roots,

$$
F_{n-2}(\theta)=R \sum_{k=0}^{n-2} \bar{\gamma}_{k}^{*} e^{i k \theta}=\sum_{k=0}^{n-2}\left|\gamma_{k}^{*}\right| \cos \left(k \theta-\alpha_{k}\right),
$$

where $\alpha_{k}=\arg \gamma_{k}^{*},(k=0,1,2, \cdots, n-2)$, and $\alpha_{0}=0$.

On putting $\alpha=\left(\theta_{1}+\theta_{2}\right) / 2, \delta=\left(\theta_{1}-\theta_{2}\right) / 2$, we see easily that

$$
\begin{aligned}
\gamma_{0}= & \gamma_{0}^{*}\left(1+\frac{1}{2} \cos 2 \delta\right)-\left|\gamma_{1}^{*}\right| \cos \left(\alpha_{1}-\alpha\right) \cos \delta \\
& +\frac{1}{4}\left|\gamma_{2}^{*}\right| \cos \left(\alpha_{2}-2 \alpha\right) ; \quad\left|\gamma_{n}\right|=\frac{1}{4}\left|\gamma_{n-2}^{*}\right| .
\end{aligned}
$$

We see that $\left|\gamma_{n}\right|$ does not depend on $\alpha$, nor on $\delta$; on the other hand $\gamma_{0}$ is maximal for $\delta=0$ if $\cos \left(\alpha_{1}-\alpha\right) \leqq 0$, or for $\delta=\pi$ if $\cos \left(\alpha_{1}-\alpha\right) \geqq 0$. In both cases the minimal value of $\left|\gamma_{n}\right|$ under condition $\gamma_{0}=1$ corresponds to $\delta=0$ or $\delta=\pi$; therefore $\theta_{1}$ and $\theta_{2}$ coincide. $\ddagger$

3. Polynomials for which $\gamma_{n}$ has Extremal Values. It follows from this lemma that $G_{n}^{*}(\theta)$ is

$$
G_{n}^{*}(\theta)=C[1+\cos (\theta+\alpha)]^{n}
$$

$\alpha$ being an arbitrary real argument; it may be written thus:§

$$
G_{n}^{*}(\theta)=\frac{C}{2^{n-1}}\left\{\frac{1}{2} C_{2 n, n}+\sum_{k=1}^{n} C_{2 n, n-k} \cos k(\theta+\alpha)\right\} .
$$

$\dagger$ It is clear that all real roots of a non-negative trigonometric polynomial are of even multiplicity.

$\ddagger \theta_{1}$ and $\theta_{1}+2 \pi$ are not considered as different.

$\S$ See [6], p. 392 . 
For this polynomial we have

$$
\gamma_{0}=\frac{1}{2^{n}} C\left(C_{2 n, n}\right) ; \quad\left|\gamma_{n}\right|=\frac{1}{2^{n-1}} C,
$$

whence we find the ratio

$$
\frac{\left|\gamma_{n}\right|}{\gamma_{0}}=\frac{2}{C_{2 n, n}} .
$$

We have proved the following theorem.

THEOREM 1. If $G_{n}(\theta)$ is a non-negative trigonometric polynomial,

$$
G_{n}(\theta)=1+R \sum_{k=1}^{n} \bar{\gamma}_{k} e^{i k \theta},
$$

of order $n$ with real roots, then

$$
\frac{2}{C_{2 n, n}} \leqq\left|\gamma_{n}\right| \leqq 1
$$

the maximum is attained for the polynomial $\dagger$

$$
G_{\max }(\theta)=1+\cos n(\theta+\alpha),
$$

and the minimum for the polynomial

$$
G_{\min }(\theta)=\frac{2^{n}}{C_{2 n, n}}\{1+\cos (\theta+\alpha)\}^{n},
$$

$\alpha$ being an arbitrary real argument.

4. The Generalized Extremal Problem. Consider now the following extremal problem.

Problem 2. Find the minimum of the ratio

$$
\frac{A_{m}^{2}+B_{m}^{2}}{\lambda A_{0}^{2}+\sum_{k=1}^{m}\left(A_{k}^{2}+B_{k}^{2}\right)},
$$

† See [1], [2]. 
where

$g_{m}(\theta)=A_{0}+A_{1} \cos \theta+B_{1} \sin \theta+\cdots+A_{m} \cos m \theta+B_{m} \sin m \theta$ is a trigonometric polynomial of order $m$ with real roots, and $\lambda$ is an arbitrary non-negative number.

The above mentioned problem of Blumenthal [6] corresponds to $\lambda=1$. It is easy to see that for $\lambda=2$ Problem 2 is a particular case of the Problem 1. Indeed we see that

$$
g_{m}^{2}(\theta)=G_{n}(\theta)=R \sum_{k=0}^{n} \bar{\gamma}_{k} e^{i k \theta}
$$

is a non-negative trigonometric polynomial of order $n=2 m$, while

(14) $\gamma_{0}=A_{0}^{2}+\frac{1}{2} \sum_{k=1}^{m}\left(A_{k}^{2}+B_{k}^{2}\right), \quad\left|\gamma_{n}\right|=\frac{A_{m}^{2}+B_{m}^{2}}{2}$;

therefore we have for $\lambda=2$

$$
\frac{A_{m}^{2}+B_{m}^{2}}{2 A_{0}^{2}+\sum_{k=1}^{m}\left(A_{k}^{2}+B_{k}^{2}\right)} \geqq \frac{2}{C_{2 n, n}}=\frac{2}{C_{4 m, 2 m}} .
$$

To solve our problem for all $\lambda \geqq 0$ we shall put it in the following form.

Problem 2'. Find the maximum of the expression

$$
\begin{aligned}
L(G)=\frac{1}{2 \pi} \int_{0}^{2 \pi} G_{n}(\theta) d \theta+\epsilon\left\{\frac{1}{2 \pi} \int_{0}^{2 \pi}\left[G_{n}(\theta)\right]^{1 / 2} d \theta\right\}^{2}, & \\
& (\epsilon \geqq-1),
\end{aligned}
$$

where $G_{n}(\theta)$ is a non-negative trigonometric polynomial

$$
G_{n}(\theta)=R \sum_{k=0}^{n} \bar{\gamma}_{k} e^{i k \theta}, \quad\left(\left|\gamma_{n}\right|=1\right),
$$

of order $n=2 m$ with real roots.

5. Equality of Roots of $G_{n}^{*}(\theta)$ for Problem $2^{\prime}$. We shall prove the following lemma. 
LEMma 2. All roots of the polynomial $G_{n}^{*}(\theta)$ for which the maximum in Problem $2^{\prime}$ is attained must be equal.

Put

$$
\begin{aligned}
{\left[G_{n}(\theta)\right]^{1 / 2} } & =2 \sin \frac{\theta-\theta_{1}}{2} \sin \frac{\theta-\theta_{2}}{2} F_{m-1}(\theta) \\
& =[\cos \delta-\cos (\theta-\alpha)] F_{m-1}(\theta),
\end{aligned}
$$

where $\alpha=\left(\theta_{1}+\theta_{2}\right) / 2, \delta=\left(\theta_{1}-\theta_{2}\right) / 2$, and $F_{m-1}(\theta)$ is a non-negative trigonometric polynomial of order $m-1$,

$$
F_{m-1}(\theta)=R \sum_{k=0}^{m-1} \bar{c}_{k} e^{i k \theta}
$$

with real roots. Thus we get

$$
\text { (19) } \frac{1}{2 \pi} \int_{0}^{2 \pi}\left[G_{n}(\theta)\right]^{1 / 2} d \theta=c_{0} \cos \delta-\frac{1}{2}\left|c_{1}\right| \cos \left(\beta_{1}-\alpha\right) \text {, }
$$

where $\beta_{k}=\arg c_{k},(k=0,1, \cdots, m-1)$, and $\beta_{0}=0$. Further let

then we obtain

$$
F_{m-1}^{2}(\theta)=R \sum_{k=0}^{2 m-2} \bar{c}_{k}^{*} e^{i k \theta}
$$

$$
\begin{aligned}
& \frac{1}{2 \pi} \int_{0}^{2 \pi} G_{n}(\theta) d \theta=c_{0}^{*}\left(1+\frac{1}{2} \cos 2 \delta\right) \\
& -\left|c_{1}^{*}\right| \cos \left(\beta_{1}^{*}-\alpha\right) \cos \delta+\frac{1}{4}\left|c_{2}^{*}\right| \cos \left(\beta_{2}^{*}-2 \alpha\right),
\end{aligned}
$$

where $\beta_{k}^{*}=\arg c_{k}^{*},(k=0,1, \cdots, 2 m-2)$, and $\beta_{0}^{*}=0$. Using (19) and (21) we have

$$
L(G)=A \cos 2 \delta+B \cos \delta+C,
$$

where

$$
\begin{aligned}
A= & \frac{1}{2}\left(c_{0}^{*}+\epsilon c_{0}^{2}\right), \\
B= & -\left|c_{1}^{*}\right| \cos \left(\beta_{1}^{*}-\alpha\right)-\epsilon c_{0}\left|c_{1}\right| \cos \left(\beta_{1}-\alpha\right), \\
C= & \frac{1}{4}\left|c_{2}^{*}\right| \cos \left(\beta_{2}^{*}-2 \alpha\right) \\
& +\frac{1}{4} \epsilon\left|c_{1}\right|^{2} \cos ^{2}\left(\beta_{1}-\alpha\right)+c_{0}^{*}+\frac{1}{2} \epsilon c_{0}^{2} .
\end{aligned}
$$


It is important to point out that $\left|\gamma_{n}\right|=(1 / 4)\left|c_{2 m-2}^{*}\right|$ does not depend on $\alpha$, nor on $\delta$. Since we have

$$
c_{0}^{*}=c_{0}^{2}+\frac{1}{2} \sum_{k=1}^{m-1}\left|c_{k}\right|^{2}
$$

it is clear that for $\epsilon \geqq-1$ we have

$$
A=\frac{1}{2}(1+\epsilon) c_{0}^{2}+\frac{1}{4} \sum_{k=1}^{m-1}\left|c_{k}\right|^{2}>0 .
$$

Therefore $L\left(G_{n}\right)$ is maximal for $\delta=0$ if $B \geqq 0$, and for $\delta=\pi$ if $B \leqq 0$; in both cases $\theta_{1}$ and $\theta_{2}$ coincide, which proves our lemma.

6. Polynomials having the Extremal Property. We see that the polynomial $G_{n}^{*}(\theta)$ is

$$
\begin{aligned}
G_{n}^{*}(\theta) & =2^{n-1}[1+\cos (\theta+\alpha)]^{n} \\
& =\frac{1}{2} C_{2 n, n}+\sum_{k=1}^{n} C_{2 n, n-k} \cos k(\theta+\alpha),
\end{aligned}
$$

and we have for it

$$
L\left(G_{n}^{*}\right)=\frac{1}{2}\left(C_{2 n, n}+\epsilon\left(C_{n, n / 2}\right)^{2}\right) .
$$

Thus we have proved the following theorem.

TheOREM 2. If $G_{n}(\theta)$ is a non-negative trigonometric polynomial of order $n=2 m$,

$$
G_{n}(\theta)=R \sum_{k=0}^{n} \bar{\gamma}_{k} e^{i k \theta}, \quad\left|\gamma_{n}\right|=1,
$$

with real roots, then

$$
\begin{aligned}
\frac{1}{2 \pi} \int_{0}^{2 \pi} G_{n}(\theta) d \theta & +\epsilon\left\{\frac{1}{2 \pi} \int_{0}^{2 \pi}\left[G_{n}(\theta)\right]^{1 / 2} d \theta\right\}^{2} \\
& \leqq \frac{1}{2}\left(C_{2 n, n}+\epsilon\left(C_{n, n / 2}\right)^{2}\right), \quad(\epsilon \geqq-1)
\end{aligned}
$$

the maximum is attained for the polynomial 


$$
G_{n}^{*}(\theta)=2^{n-1}\{1+\cos (\theta+\alpha)\}^{n},
$$

$\alpha$ being an arbitrary real argument.

This result may also be stated as the following theorem.

TheOREM $2^{\prime}$. If $g_{m}(\theta)$ is a trigonometric polynomial of order $m$, $g_{m}(\theta)=A_{0}+A_{1} \cos \theta+B_{1} \sin \theta+\cdots+A_{m} \cos m \theta+B_{m} \sin m \theta$, with real roots, then

$$
\frac{A_{m}^{2}+B_{m}{ }^{2}}{\lambda A_{0}{ }^{2}+\sum_{k=1}^{m}\left(A_{k}{ }^{2}+B_{k}{ }^{2}\right)} \geqq \frac{2}{C_{4 m, 2 m}+\frac{\lambda-2}{2}\left(C_{2 m, m}\right)^{2}}, \quad(\lambda \geqq 0) ;
$$

this minimum is attained for the polynomial

$$
g_{m}^{*}(\theta)=C\{1+\cos (\theta+\alpha)\}^{m} .
$$

\section{BIBLIOGRAPHY}

1. L. Fejér, Über trigonometrische Polynome, Journal für Mathematik, vol. 146 (1915), pp. 53-82.

2. O. Szász, Über nichtnegative trigonometrische Polynome, Sitzungsberichte der Bayerischen Akademie der Wissenschaften, (1917), pp. 307-320.

3. O. Szász, Elementare Extremalprobleme über nichtnegative trigonometrische Polynome, ibid., (1927), pp. 185-196.

4. O. Szász, Über harmonische Funktionen und L-Formen, Mathematische Zeitschrift, vol. 1 (1918), pp. 149-162.

5. E. v. Egerváry und O. Szász, Einige Extremalprobleme im Bereiche der trigonometrischen Polynome, ibid., vol. 27 (1927), pp. 641-652.

6. O. Blumenthal, Einige Minimums-Sätze über trigonometrische und rationale Polynome, Mathematische Annalen, vol. 77 (1916), pp. 390-403.

Mathematical Institute,

KHARKOW, U.S.S.R. 